\title{
Emergent community agglomeration from data set geometry
}

\author{
Chenchao Zhao \\ Department of Physics and Carl R. Woese Institute for Genomic Biology, University of Illinois at Urbana-Champaign, \\ Urbana, Illinois 61801, USA \\ Jun S. Song* \\ Department of Physics, Carl R. Woese Institute for Genomic Biology, \\ and Department of Bioengineering, University of Illinois at Urbana-Champaign, Urbana, Illinois 61801, USA
}

(Received 1 January 2017; revised manuscript received 20 March 2017; published 13 April 2017)

\begin{abstract}
In the statistical learning language, samples are snapshots of random vectors drawn from some unknown distribution. Such vectors usually reside in a high-dimensional Euclidean space, and thus the "curse of dimensionality" often undermines the power of learning methods, including community detection and clustering algorithms, that rely on Euclidean geometry. This paper presents the idea of effective dissimilarity transformation (EDT) on empirical dissimilarity hyperspheres and studies its effects using synthetic and gene expression data sets. Iterating the EDT turns a static data distribution into a dynamical process purely driven by the empirical data set geometry and adaptively ameliorates the curse of dimensionality, partly through changing the topology of a Euclidean feature space $\mathbb{R}^{n}$ into a compact hypersphere $S^{n}$. The EDT often improves the performance of hierarchical clustering via the automatic grouping information emerging from global interactions of data points. The EDT is not restricted to hierarchical clustering, and other learning methods based on pairwise dissimilarity should also benefit from the many desirable properties of EDT.
\end{abstract}

DOI: 10.1103/PhysRevE.95.042307

\section{INTRODUCTION}

Community detection, better known as clustering in the literature of statistical learning [1-7], is a process of merging similar nodes of a complex network into communities (clusters) and often shows a hierarchical organization of communities at different levels of similarity. Akin to the idea of renormalization group in physics, decreasing the threshold for similarity leads to increasingly coarse-grained pictures of the "microscopic" network. The reduction in complexity can sometimes yield more interpretable statistical models that could serve as a basis for further classification analysis. Along this line, we present an idea of transforming dissimilarity measures to allow dynamic agglomeration of data points into communities.

Complexity in networks is analogous to that in many-body systems. Thus clustering algorithms based on classical spin models have been designed by statistical physicists; e.g., each data point is replaced by a spin, and the similarity between points is computed from their Euclidean distance and spin orientations [8,9]. Although such algorithms are both applicable and theoretically interesting, they usually require intensive Monte Carlo simulations and are thus too complex to implement in practical data analysis compared to other popular deterministic clustering algorithms. With practicality in mind, we present a nonlinear transformation of data set geometry and then pass the transformed geometric

\footnotetext{
*songj@illinois.edu
}

Published by the American Physical Society under the terms of the Creative Commons Attribution 4.0 International license. Further distribution of this work must maintain attribution to the author(s) and the published article's title, journal citation, and DOI. information to the standard hierarchical clustering algorithm widely used in contemporary data analysis. We show that the geometric transformation effectively captures a collective interaction among all sample points and that such global network interaction often improves the accuracy of community detection.

Most statistical learning algorithms utilize a pairwise dissimilarity measure $d_{i j}^{(0)}$ that depends only on the $(i, j)$ pair of samples. In the context of Einstein's theory of gravity, or Riemannian geometry, the "geometry" is completely encoded in the metric tensor. This paper adopts the same notion of geometry for a data set and focuses on the information encoded in the dissimilarities between all pairs of sample points. The $n$ features of $m$ samples measured in an experiment are typically organized into an $n \times m$ matrix, with $m$ samples represented as points in $\mathbb{R}^{n}$. Thus the Euclidean $L^{p}$ metric directly defined on the feature space $\mathbb{R}^{n}$ is among the most common pairwise dissimilarities.

In high dimensions, however, the relative contrast between the farthest and nearest points measured by the $L^{p}$-metric diminishes; consequently, the concept of nearest neighbors, which serves as the foundation for clustering, becomes increasingly ill-defined as the feature dimension increases [10-12]. This phenomenon is termed "the curse of dimensionality," analogous to the idea of "more is different" for many-body systems [13]. Modifications of Euclidean distances are found to improve the relative contrast for an artificial data cloud drawn from a single distribution [10,11], but fail in data drawn from several distributions [12]. One way to address the loss of contrast in high dimensions for multidistribution data is to introduce an effective dissimilarity measure calculated from the number of shared nearest neighbors of two data points, where each point is allowed to have a fixed number of nearest neighbors [12]. The use of effective dissimilarity reduces the effect of high feature dimensions in subsequent computations; 
however, the choice of effective dissimilarity function actually dictates the improvement.

This paper proposes an effective dissimilarity transformation (EDT), where all data points in the primary feature space participate in redefining the effective dissimilarity between any two given data points. Our main motivation stems from the empirical formula for distance correlation in statistics [14] and the idea of heat flow on a hypersphere in support vector machine classification $[15,16]$. Empirical distance correlation utilizes the covariance of pairwise distance between all samples to measure statistical association between two random vectors [14]. In this spirit, our EDT can be viewed as measuring the similarity between two data points by taking a dot product of the corresponding columns of uncentered distance matrix. More precisely, transforming data to lie on a hypersphere has been previously shown to yield several advantages in machine learning $[15,16]$, so an intermediate step in EDT maps the columns of distance matrix to points on a hypersphere before taking a dot product. We show that this simple EDT improves the contrast between clusters in a geometrically interpretable manner and that it is able to reshape a geometrically mixed data distribution into separable clusters.

To be specific, the effective dissimilarity obtained from EDT is beyond the pairwise level and globally captures relations to all available sample points. Moreover, we also observe that the EDT is a map defined on a non-negative dissimilarity space $\mathbb{R}_{\geqslant 0}^{m}$ of samples, where typically the sample size $m$ is much smaller than the feature dimension $n$, thus providing an efficient dimensional reduction scheme. Iteratively applying the transformation yields a sequence $d_{i j}^{(\tau)}$ of EDT parametrized by a non-negative integer $\tau$. As $\tau$ increases, microscopic structures condense locally, while intercluster macroscopic distinctions become more evident. Since the heat kernels describing heat diffused from a point source is parametrized by continuous time $t \geqslant 0$, we may interpret EDT as a generalized nonlinear diffusion process in the dissimilarity space driven by the distribution of samples. Iterating EDT thus turns a static distribution of points into a dynamical process and often amplifies its power of cluster separation.

\section{RESULTS}

\section{A. Formulation of effective dissimilarity transformation (EDT)}

As observed in previous support vector machine (SVM) classification studies $[15,16]$, hyperspherical geometry often improves classification accuracy. Motivated by these results, we now introduce an effective dissimilarity transformation based on a hyperspherical representation of data clouds. To map sample points onto a hypersphere, we will utilize the following hyperspherical transformation from non-negative space $\mathbb{R}_{\geqslant 0}^{m} \backslash\{0\}$ to a unit hypersphere.

Definition 1. A hyperspherical projective map $\varphi: \mathbb{R}_{\geqslant 0}^{m} \backslash$ $\{0\} \rightarrow S^{m-1}$ maps a vector $\mathbf{x}$, with $x_{i} \geqslant 0$ and $\sum_{i=1}^{m} x_{i}>0$, to a unit vector $\hat{x} \in S^{m-1}$ where $(\hat{x})_{i} \equiv \sqrt{x_{i} / \sum_{j=1}^{m} x_{j}}$.

A useful measure of similarity on a hypersphere is the cosine similarity.

Definition 2. For unit vectors $\hat{x}=\varphi(\mathbf{x})$ and $\hat{y}=\varphi(\mathbf{y})$ obtained from non-negative vectors $\mathbf{x}, \mathbf{y} \in \mathbb{R}_{\geqslant 0}^{m} \backslash\{0\}$ via the hyperspherical projective map, the cosine similarity is the dot product $\hat{x} \cdot \hat{y}$.

The EDT relies on this notion of cosine similarity, as explained below.

Many algorithms - such as hierarchical clustering, KMedoids, and KMeans-directly rely on some notion of difference between samples. For example, the Euclidean distance function is a popular measure of the difference between two sample points in $\mathbb{R}^{n}$. In statistical learning approaches based on pairwise differences, however, we often relax the definiteness condition and triangular inequality satisfied by a distance function and utilize instead a more general and flexible measure of difference, called the dissimilarity function.

Definition 3. A dissimilarity function defined on a manifold $\mathcal{M}$ is a map $d: \mathcal{M} \times \mathcal{M} \rightarrow \mathbb{R}_{\geqslant 0}$ satisfying (1) nonnegativity: $d(x, y) \geqslant 0$ for all $x, y \in \mathcal{M}$; (2) identity: $d(x, x)=0$ for all $x \in \mathcal{M}$; (3) symmetry: $d(x, y)=d(y, x)$ for all $x, y \in \mathcal{M}$.

Usually $\mathcal{M}=\mathbb{R}^{n}$, representing the sample space of original data directly collected from experiments, and its nonlinear embedding into an abstract manifold is often only implicitly defined through the dissimilarity function.

Dissimilarity functions are relatively easy to construct; in particular, we can turn the cosine similarity on $\mathbb{R}_{\geqslant 0}^{n} \backslash\{0\}$ into a dissimilarity function by defining $d(\mathbf{x}, \mathbf{y})=1-\hat{x}$. $\hat{y}=\frac{1}{2}\|\hat{x}-\hat{y}\|^{2}$. We here show that this cosine dissimilarity function can be iteratively applied to an initial dissimilarity measure and that this simple iteration leads to several robust properties desirable for clustering applications.

More precisely, given an initial dissimilarity function $d(\cdot, \cdot)$ and $m$ sample points, organize the pairwise dissimilarity of the samples into an $m \times m$ non-negative, symmetric dissimilarity matrix $d^{(0)}$. To apply our method, we only need to assume the mild condition that each column of $d^{(0)}$ is not a zero vector. We then define the effective dissimilarity transformation on the space of such matrices as follows.

Definition 4. The effective dissimilarity transformation (EDT) $\psi: \mathbb{R}_{\geqslant 0}^{m \times m} \rightarrow \mathbb{R}_{\geqslant 0}^{m \times m}$ is defined as

$$
\left[\psi\left(d^{(0)}\right)\right]_{i j}=\frac{1}{2}\left\|\varphi\left(\mathbf{p}_{i}\right)-\varphi\left(\mathbf{p}_{j}\right)\right\|^{2},
$$

where $\mathbf{p}_{i}$ is the $i$ th column of the dissimilarity matrix $d^{(0)}$ and $\varphi$ is the hyperspherical projective map into $S^{m-1}$. We denote $d^{(1)} \equiv \psi\left(d^{(0)}\right)$.

The resulting $d^{(1)}$ is thus a cosine dissimilarity matrix of the $m$ samples represented by the columns of the dissimilarity matrix $d^{(0)}$. Importantly, the pairwise dissimilarity captured by $d^{(1)}$ between any two samples measures how dissimilar are their respective $d^{(0)}$ dissimilarities to all samples; in other words, each entry of $d^{(1)}$ depends on the global network structure encoded in $d^{(0)}$ as illustrated in Fig. 1(a). Iterating the map composition $\psi^{(\tau+1)}=\psi \circ \psi^{(\tau)}$ yields a sequence $\left\{\psi^{(\tau)}\right\}_{\tau=0}^{\infty}$ of EDTs and corresponding dissimilarity matrices $\left\{d^{(\tau)}\right\}_{\tau=0}^{\infty}$, where $\psi^{(0)}$ is the identity map and $d^{(\tau)}=\psi^{(\tau)}\left(d^{(0)}\right)$. The sequence of dissimilarity matrices $\left\{d^{(\tau)}\right\}_{\tau=0}^{\infty}$ may be interpreted as inducing a data-driven evolution or flow of sample points parametrized by $\tau$. This paper shows that the data-driven redefinition of dissimilarity resulting from an iterated application of EDT often leads to improved clustering results. 
(a)

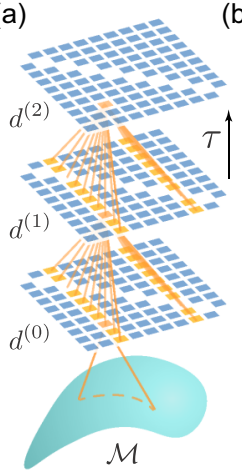

(b)

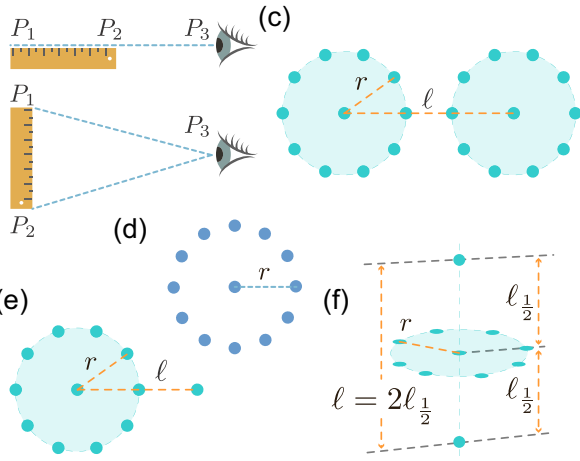

FIG. 1. (a) Schematic illustration of the network structure of effective dissimilarity transformations (EDT) parametrized by $\tau$. The $(i, j)$ th entry of $d^{(\tau)}$ arises from transforming the $i$ th and $j$ th columns of $d^{(\tau-1)}$. (b) Illustrations of perspective contraction effect of EDT. (c) Two ideal clusters with radius $r$ and centroid-centroid distance $\ell$ in $\mathbb{R}^{2}$. (d) The detector used in the measurement of local deformation of data distributions in $\mathbb{R}^{2}$. (e) An ideal cluster of radius $r$ in $\mathbb{R}^{2}$ and an outlier at distance $\ell$ from the cluster centroid. (f) An ideal cluster of radius $r$ in the $x y$ plane of $\mathbb{R}^{3}$ with symmetrically located outliers on the $z$ axis at distance $\ell_{\frac{1}{2}}=\frac{\ell}{2}$ from cluster centroid.

Even though EDT is simple in its definition and deterministic in nature, its nonlinearity makes the flow of data points difficult to study. Consequently, we first study the properties of EDT by performing Gedankenexperimente on carefully designed synthetic data sets shown in Figs. 1(b)-1(f) [accompanying simulation results in Figs. 2(a)-2(f)], and then test the power of these observed properties in the setting of real data sets.

\section{B. Gedankenexperimente of EDT}

First consider the simple data set consisting of three distinct points, $P_{1}, P_{2}$, and $P_{3}$, in $\mathbb{R}^{n}$, for any $n \geqslant 2$. Let $P_{1}$ and $P_{2}$ represent two ends of a ruler of length $d_{12}^{(0)}=a$, and let $P_{3}$ represent an observer at distance $b$ to the center of the ruler; Fig. 1(b) shows two particular cases: (1) the ruler and
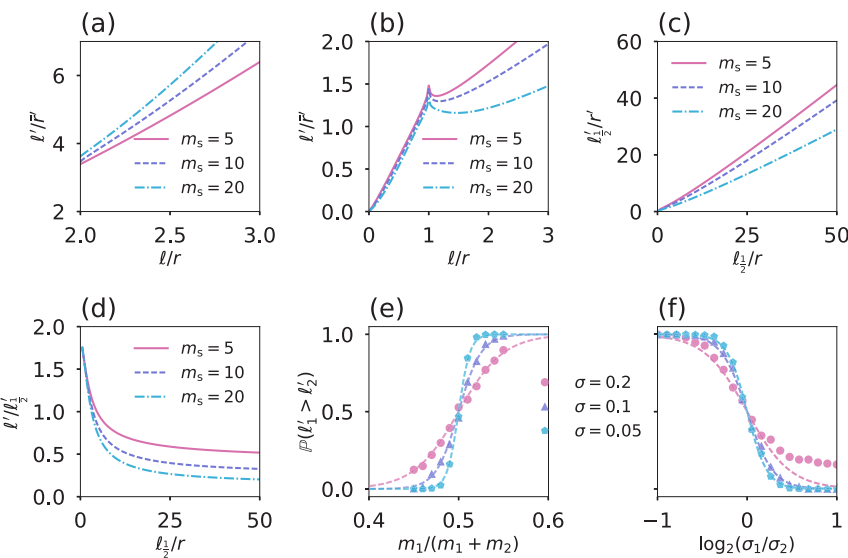

FIG. 2. Results of Gedankenexperimente: (a) cluster condensation [Fig. 1(c)], (b) single outlier absorption [Fig. 1(e)], (c),(d) two outliers perpendicular to an ideal cluster [Fig. 1(f)], and (e),(f) probabilistic sampling.
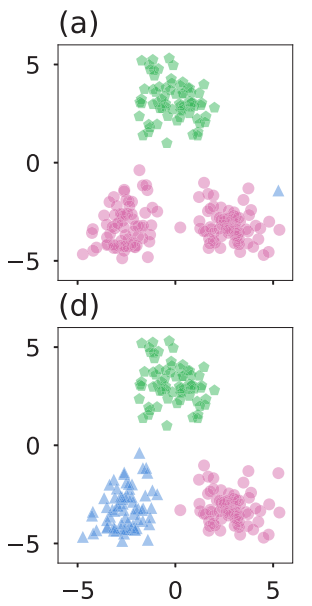

(b)

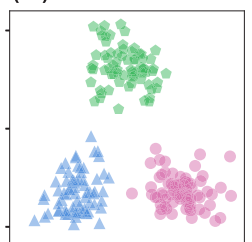

(e)

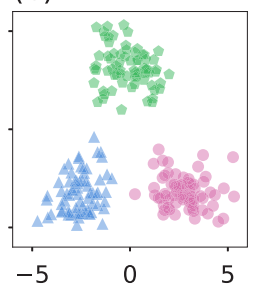

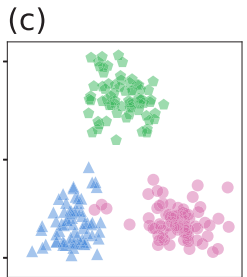

(f)

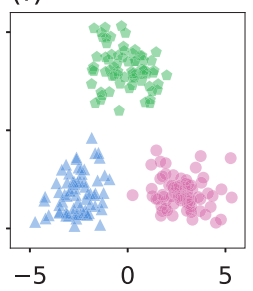

FIG. 3. Comparison of hierarchical clustering results using (a)-(c) Euclidean distance vs (d)-(f) EDT-enhanced Euclidean distance with (a),(d) single, (b),(e) average, and (c),(f) complete linkages. The number of clusters was chosen to be three in the analysis.

observer are collinear, and $b>a / 2$; (2) the observer and ruler form an isosceles triangle, and $d_{23}^{(0)}=d_{13}^{(0)}=c=\sqrt{\left(\frac{a}{2}\right)^{2}+b^{2}}$. In scenario (1), the original distance $d_{12}^{(0)}$ between $P_{1}$ and $P_{2}$ is equal to the ruler length and is also the observed distance $d_{13}^{(0)}-d_{23}^{(0)}$ measured by the observer at $P_{3}$, irrespective of the location of $P_{3}$; after EDT, however, both $d_{12}^{(1)}$ and the ratio $\left(d_{13}^{(1)}-d_{23}^{(1)}\right) / d_{12}^{(1)}=\sqrt{\frac{a}{2 b}}$ shrink as the observer moves away (Appendix B 1). That is, in the limit $b \gg a$, the effective dissimilarity between $P_{1}$ and $P_{2}$ approaches zero, and the observer at $P_{3}$ cannot distinguish between $P_{1}$ and $P_{2}$ on the scale set by $d_{12}^{(1)}$. In the language of hierarchical clustering, the single, average, and complete linkages become equivalent after EDT as $P_{3}$ becomes a clear outgroup. Similarly, in scenario (2), the effective ruler length also shrinks as the observer moves away from the other two points, i.e., $d_{12}^{(1)}=1-\frac{c}{a+c} \downarrow 0$ as $b / a \uparrow \infty$. We can thus summarize these properties as a perspective contraction effect.

Observation 1. The EDT dissimilarity between each pair of points shrinks as an observer moves away from the distribution of points. Consequently, compared to the original dissimilarity, hierarchical clustering using the EDT dissimilarity is insensitive to the choice of linkage.

We verified this observation by comparing the performance of Euclidean distance with its EDT dissimilarity in the hierarchical clustering of three Gaussian clouds in $\mathbb{R}^{2}$ using single, average, and complete linkages (Fig. 3). As often is the case with real data, the three linkages based on the Euclidean distance led to different clustering results (Fig. 3, top row), whereas the EDT dissimilarity was insensitive to the choice of linkage (Fig. 3, bottom row)

We next replaced the ruler and observer in our first model with two identical ideal clusters, each of which consisted of a centroid point and $m_{\mathrm{s}}$ uniformly distributed satellites at radius $d_{\mathrm{cs}}^{(0)}=r$ in $\mathbb{R}^{2}$ [Fig. 1(c)]. The distance between the two centroids was set to $d_{\mathrm{cc}}^{(0)}=\ell>2 r$, and data distribution had two global mirror reflection symmetries about (1) the line 

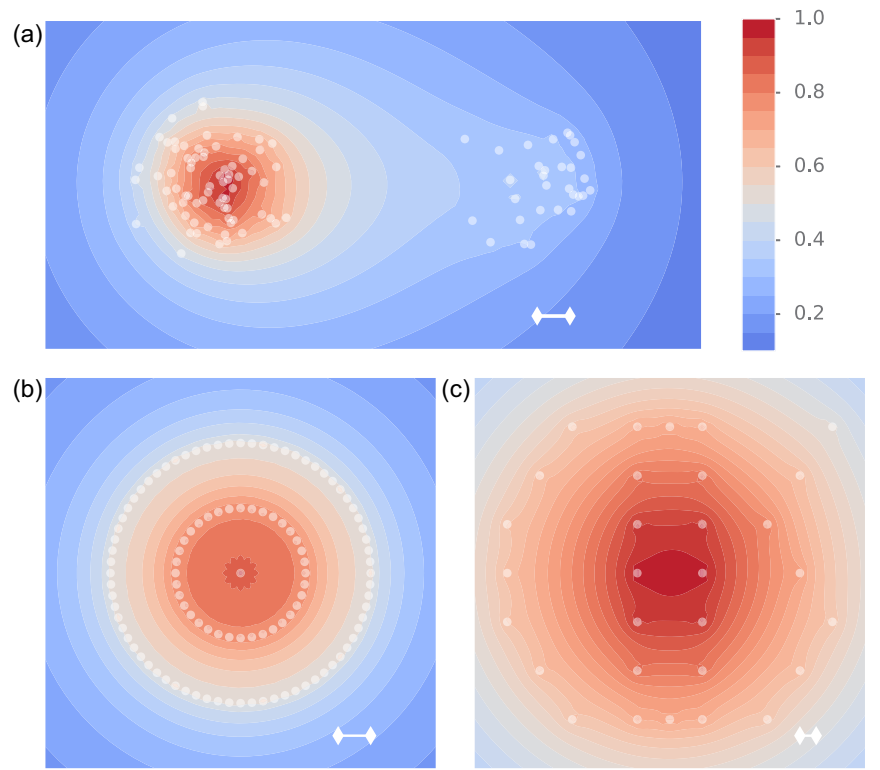

FIG. 4. $v$ distribution for three data sets: (a) two Gaussian distributions with equal variance, but different sample sizes $m_{\text {left }}=70$ and $m_{\text {right }}=30$; (b) two layers of circularly distributed points with radius $r_{\text {outer }}=2 r_{\text {inner }}$ (bottom left); (c) points distributed in the shape of the word "COS." Each $v$ distribution was normalized by dividing by its maximum; the white segment in each plot indicates the diameter of the detector used in the measurement of $v$.

connecting two centroids and (2) the perpendicular bisector thereof. We compared the changes in intra- and intercluster dissimilarities after EDT and found that the two circles were deformed, but the global mirror reflection symmetries were preserved. We further measured the mean $\bar{r}^{\prime} \equiv\left\langle d_{\mathrm{cs}}^{(1)}\right\rangle$ and $\ell^{\prime} \equiv d_{\mathrm{cc}}^{(1)}$ and found the ratio $\ell^{\prime} / \bar{r}^{\prime}$ to be an increasing function in both $\ell / r$ and $m_{\mathrm{s}}$; moreover, $\ell^{\prime} / \bar{r}^{\prime}>\ell / r$ for any $\ell>2 r$ [Fig. 2(a)]. Thus the EDT had the effect of forcing the data points in each cluster to condense towards their respective centroid location, a potentially desirable effect that can help automatically merge data points into correct communities. We summarize our observation as a cluster condensation effect.

Observation 2. For separable clusters, the EDT condenses the points within a cluster, while inflating the space between clusters; this cluster condensation effect becomes stronger with the number of points in each cluster and also with the initial intercluster dissimilarity.

The previous two Gedankenexperimente were performed on highly symmetric data sets. To probe the local deformation induced by EDT on a generic data distribution, we devised a detector, or a composite "test charge." The idea is generalizable to higher feature dimensions, but to simplify the interpretation, we performed the simulation in $\mathbb{R}^{2}$, with the detector being an ideal cluster of 12 sensor points at radius $r$ from a centroid point [Fig. 1(d)]. Deviations of the detector from a perfect circle in local ambient distributions were used to assess the EDT impact landscape. We captured the deviations through the transformed arm lengths $\left\{r_{i}^{\prime}\right\}_{i=1}^{12}$ of the 12 sensors after EDT; we then derived two scalar quantities of interest: (1) the mean arm length $v=\left\langle r_{i}^{\prime}\right\rangle$ that measures a volume change and (2) the standard deviation of $\left\{r_{i}^{\prime} / \nu\right\}_{i=1}^{m_{\mathrm{s}}}$, denoted
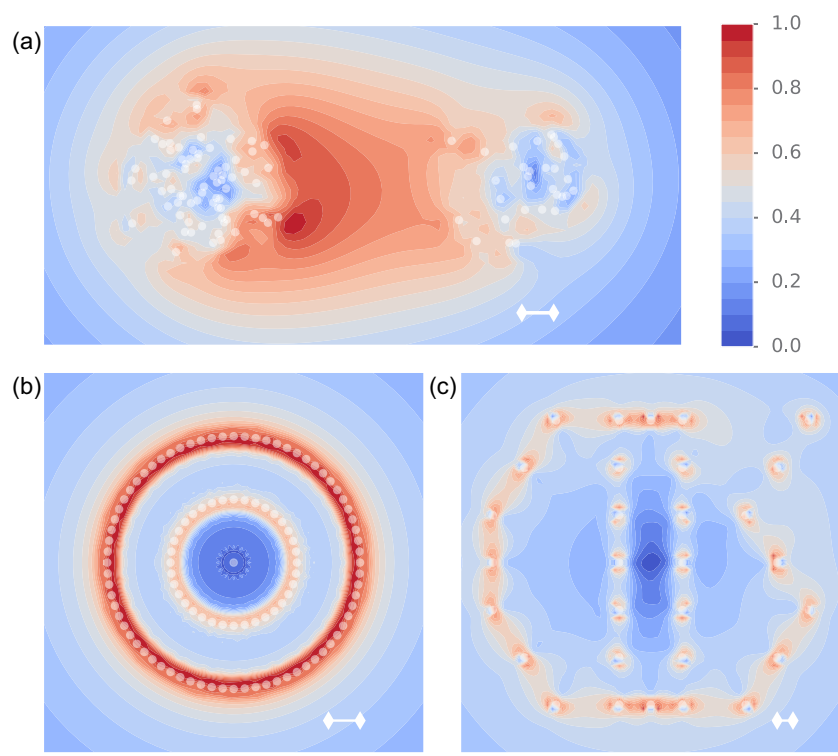

FIG. 5. $\kappa$ distribution for three data sets: (a) two Gaussian distributions with equal variance, but different sample sizes $m_{\text {left }}=70$ and $m_{\text {right }}=30$; (b) two layers of circularly distributed points with radius $r_{\text {outer }}=2 r_{\text {inner }}$; (c) points distributed in the shape of the word "COS." Each $\kappa$ distribution was normalized by dividing by its maximum; the white segment in each plot indicates the diameter of the detector used in the measurement of $\kappa$.

$\kappa$, that measures anisotropy or the effect of "tidal force" from probed data points. The observed volume changes were consistent with the effect of "perspective contraction," and the mean arm length $v$ of the detector shrank as it moved away from high density regions of the probed data distribution (Fig. 4). The $\kappa$ distributions were highly nontrivial, as illustrated in Fig. 5: $\kappa$ attained high values whenever the rim of the detector was near a data point, indicating an intense tug-of-war between the data points and the detector that were both trying to capture the sensors; by contrast, the normalized $\kappa$ almost vanished at the centers of two Gaussian distributions, within the inner circle of the two layers of circularly distributed points, and at the center of "O" in the "COS" data. The low values of $\kappa$ in the interior of clustered data suggest a screening effect that shields the interior from anisotropic distortions, akin to the shielding effect of conductors in electrostatics; this effect may potentially protect subcluster structures within a dense cluster.

Inspired by the high values of $\kappa$ near the boundary of a cluster, we performed additional experiments to test the effect of EDT on outliers, using (1) an ideal cluster in $\mathbb{R}^{2}$ with $m_{\mathrm{s}}$ satellites at radius $r$ from the center point and an additional single point at varying distance $\ell$ from the center [Fig. 1(e)] and (2) the same ideal cluster in the $x y$ plane of $\mathbb{R}^{3}$ and two outliers located on the $z$ axis at $z= \pm \ell / 2$ [Fig. 1(f)]. For the first case, Fig. 6 shows how a cluster of points traps an outlier and prevents it from escaping the cluster. Furthermore, in both cases, we observed that the trapping power increased with cluster mass $m_{\mathrm{s}}$ : in case (1), increasing $m_{\mathrm{S}}$ reduced the relative effective outlier-centroid dissimilarity $\ell^{\prime} / \bar{r}^{\prime}$ and broadened the outlier region that got pulled back towards the cluster 


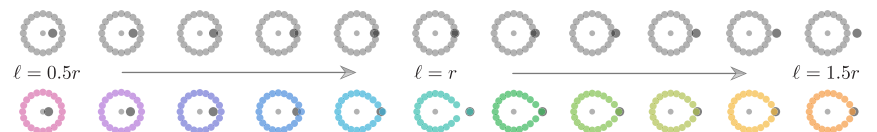

FIG. 6. Cluster of points can pull back or "trap" an outlier. Figure shows the case illustrated in Fig. 1(e) for varying values of the ratio $\ell / r$ in the range $[0.5,1.5]$ and for 20 satellite points. The top gray circles indicate the actual locations of points in $\mathbb{R}^{2}$; the bottom colored circles illustrate the corresponding effective locations after EDT, where we doubled the distortions to visualize the effect more clearly. As $\ell / r$ increased from left to right, the deformed circle behaved like an elastic membrane trying to trap the outlier from escaping and demonstrated singular behavior at $\ell=r$.

[Fig. 2(b)]; in case (2), increasing $m_{\mathrm{S}}$ also decreased the relative effective outlier-centroid dissimilarity $\ell_{\frac{1}{2}}^{\prime} / r^{\prime}$ [Fig. 2(c)]. We summarize the local deformation effect, or the "tidal force" exerted by local data distribution, as follows.

Observation 3. Under the EDT, data points deform the local distribution of neighboring points such that potential outliers tend to be trapped by a massive cluster. The deformation is strong near the exterior of a cluster and almost completely screened inside the cluster.

In case (2), we also observed an intriguing paradox: the transformed outlier-outlier dissimilarity $\ell^{\prime}$ satisfied the condition $\ell^{\prime}<2 \ell_{\frac{1}{2}}^{\prime}$ for all $\ell_{\frac{1}{2}} / r>0$, and it even satisfied the counterintuitive inequality $\ell^{\prime}<\ell_{\frac{1}{2}}^{\prime}$ for sufficiently large $\ell_{\frac{1}{2}} / r$ and large $m_{\mathrm{S}}$ [Fig. 2(d)]. A resolution of this paradox is achieved by noting that the points at infinity become identified under EDT. For example, for the particular case of circularly distributed data points in $\mathbb{R}^{2}$, as illustrated in Fig. 7, the outer rings of points become increasingly similar as $\tau$, indexing the EDT iteration, increases; moreover, the effect becomes more pronounced as the density of points at the center of the distribution increases (bottom row in Fig. 7, Appendix B 4). In mathematical terms, adding the point at infinity to $\mathbb{R}^{2}$ yields a compact sphere $S^{2}$, and the above process can be visualized as the outer rings diffusing towards the south pole (Fig. 7).

We tested whether this property of EDT can help improve clustering performance on synthetic data sets that are known to confound simple algorithms. For this purpose, we chose (a)

(e)
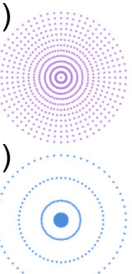

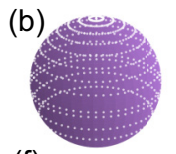

(f)

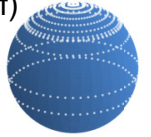

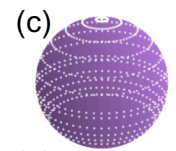

(g)

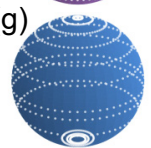

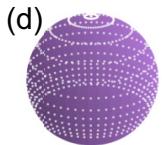

(h)
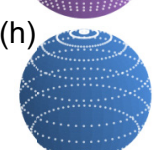

FIG. 7. EDT approximately identifies the points at infinity. We designed two uniformly circularly distributed data sets with (a) a uniform increment in radius, or (e) a small increment in radius near the center and a large increment for the outermost three circles. For both data sets, the outer circles became relatively closer as $\tau$ increased. The values $\tau=1,2$, and 3 correspond to (b),(f), (c),(g), and (d),(h), respectively. The effect was more pronounced in the case (e), and the outermost three circles were visibly mapped to the south pole. The mapping method can be found in Appendix B 4.
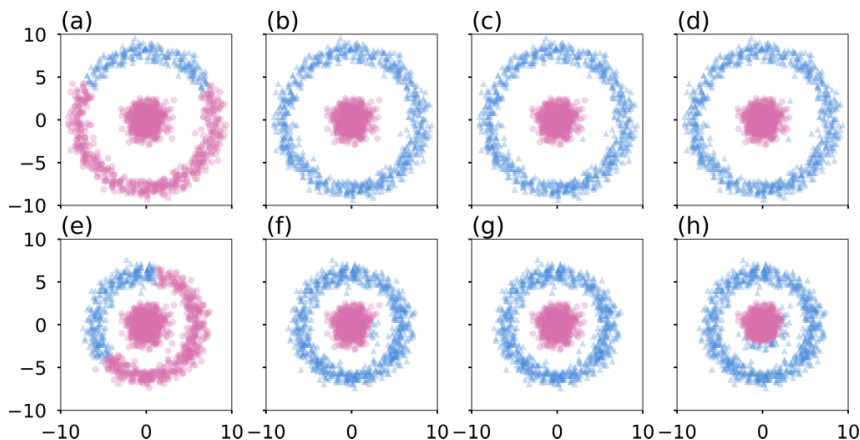

FIG. 8. Hierarchical clustering results on (a)-(d) "easy" and (e)-(h) "hard" annulus data sets using Euclidean metric $(\tau=0)$ or EDT-enhanced dissimilarities up to three iterations and using average linkage. From left to right, (a),(e), (b),(f), (c),(g), and (d),(h) correspond to $\tau=0,1,2$, and 3, respectively. Dramatic improvements were seen after just one iteration of EDT.

two clusters of data concentrically distributed with a gap in radial direction (Fig. 8). The EDT dramatically improved the performance of hierarchical clustering with Euclidean metric (Fig. 8); furthermore, the EDT-enhanced hierarchical clustering outperformed spectral clustering using Gaussian RBF as a measure of similarity (Fig. 9). These observations can be summarized as EDT's global deformation effect.

Observation 4. EDT is able to globally warp the data space on the length scale comparable to intercluster distances, such that points far from the majority distribution become approximately identified. EDT thus topologically changes $\mathbb{R}^{n}$ to $S^{n}$.

In application, the EDT will asymptotically group outliers that are very dissimilar to all clusters and may be dissimilar among themselves into one "unclassifiable" cluster in an automatic fashion.

Lastly, we considered the effect of EDT in a probabilistic sense. The initial dissimilarity $d^{(0)}$ can be thought
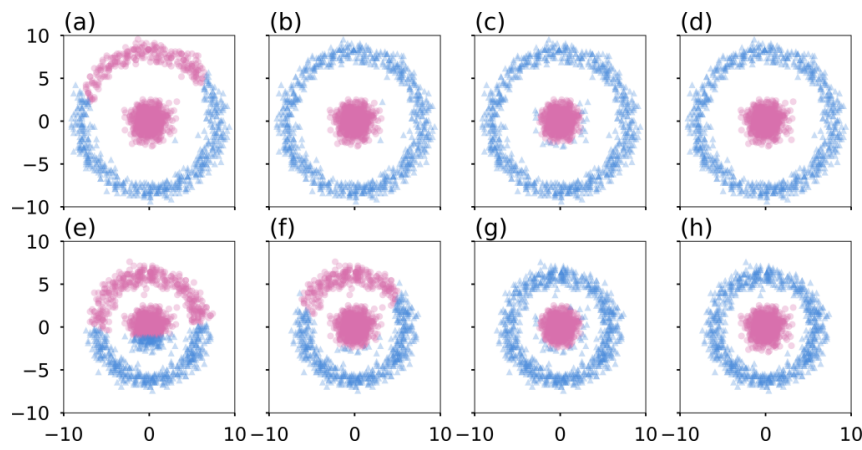

FIG. 9. Spectral clustering results for the (a)-(d) "easy" and (e)-(h) "hard" data sets from Fig. 8 using the Gaussian RBF kernel $\exp \left(-\gamma\|\mathbf{x}-\mathbf{y}\|^{2}\right)$ with $\gamma=0.05,0.1$, and 0.2 in (a),(e), (b),(f), and $(\mathrm{c}),(\mathrm{g})$, respectively, and $(\mathrm{d}),(\mathrm{h})$ the "nearest neighbors" method retaining only 10 nearest neighbors of each point to assemble a sparse similarity matrix. In SVM, nonlinear kernels with tunable hyperparameters are usually more powerful than a vanilla linear kernel $\mathbf{x} \cdot \mathbf{y}$; however, an unthresholded continuous measure of relatedness between sample points is not necessarily a blessing for unsupervised learning algorithms. 
of as a random matrix calculated from data sampled from a probability distribution. We replaced the ideal clusters in $\mathbb{R}^{2}$ in Fig. 1(c) by two independent bivariate Gaussian distributions $\mathcal{N}_{1}\left(\left(-\ell_{1}, 0\right)^{\top}, \sigma_{1}^{2}\right)$ and $\mathcal{N}_{2}\left(\left(\ell_{2}, 0\right)^{\top}, \sigma_{2}^{2}\right)$ located symmetrically about the origin, i.e., initially $\ell_{1}=\ell_{2}$. We then placed a test point at the origin and two anchor centroids at $x=-\ell_{1}$ and $x=\ell_{2}$. Denoting the transformed value of $\ell_{i}$ after one application of EDT by $\ell_{i}^{\prime}$, we used Monte Carlo simulations to compute the probability $\mathbb{P}\left(\ell_{1}^{\prime}>\ell_{2}^{\prime}\right)$, which may be viewed as the probability that the test point is clustered with $\mathcal{N}_{2}$. We performed the calculation in two different settings: (1) $\mathcal{N}_{1}$ and $\mathcal{N}_{2}$ have same number of samples $\left(m_{1}=m_{2}\right)$, but different variances; (2) $\mathcal{N}_{1}$ and $\mathcal{N}_{2}$ share the same variance $\left(\sigma_{1}^{2}=\sigma_{2}^{2}\right)$, but different number of samples. We found that the test point was more likely to join (1) a cluster drawn from the distribution with larger variance, consistent with the local deformation effect that absorbs an outlier near the boundary of a cluster into the cluster, or (2) a cluster with fewer samples, consistent with the global deformation effect of EDT that makes points from the majority distribution similar to each other. More precisely, we empirically found the $\mathbb{P}\left(\ell_{1}^{\prime}>\ell_{2}^{\prime}\right)$ to be a hyperbolic tangent sigmoid function in $m_{1} /\left(m_{1}+m_{2}\right)$ and $-\log _{2}\left(\sigma_{1} / \sigma_{2}\right)$, as shown in Figs. 2(e) and 2(f).

\section{Application of EDT in two gene expression data sets}

We tested the power of EDT on two publicly available gene expression data sets: (1) 59 cancer cell lines from NCI60 in nine cancer types; (2) 116 blood cell samples in four cell types from human hematopoietic stem cell differentiation data set [17], with 4000 most variable genes in each data set as features. We performed hierarchical clustering using the first few iterations of EDT dissimilarity. We used the variation of information (VI) as a well-defined distance between two clustering results [7]; using the given cell types as the reference clustering, we optimized the threshold for cutting the dendrogram into clusters and quantified the performance of clustering with the minimum distance to reference clustering (Fig. 10).

For the NCI60 data, the original Euclidean distance $(\tau=0)$ gave minimum VI of 1.042; but, after two rounds of EDT ( $\tau=2$ ), the VI reduced by $31.7 \%$ to 0.712 (top two rows in Fig. 10). The original Euclidean distance failed to combine all leukemia (LE) cell lines, but EDT $(\tau=2,3)$ brought LE cell lines together into a single cluster. From the very beginning $(\tau=0)$, the melanoma cell lines were in a distinct single cluster except for one outlier LOXIM-VI. Among the misclassified cell lines after two iterations of EDT, the LOXIM-VI found itself more similar to the mixture cluster of central nervous system (CNS) and breast cancer (BR) cell lines; the result is consistent with the fact that LOXIM-VI is a desmoplastic melanoma cell line and is biologically similar to neurofibroma [18].

For the blood cell data, the original Euclidean distance split the erythrocyte (Ek, where larger values of $k$ indicate latter stages of of maturity) samples into several small subclusters, and the VI was 0.706 (bottom two rows in Fig. 10). After one iteration of EDT, the VI reduced by $54.0 \%$ to 0.325 , and all $\mathrm{E} k$ samples were grouped into a single cluster with two branches-immature red blood cells (E1, E2) and more mature blood cells (E3, E4, E5)—well separated from the immune cells (T-cells, B-cells, and, natural killer cells). These results support that the EDT can help improve clustering performance in real data analysis.

\section{DISCUSSION}

In this paper, we have developed the notion of effective dissimilarity transformation to enhance the performance of hierarchical clustering, utilizing only the geometric information of all pairwise dissimilarities. The nonlinear transformation adjusts the dissimilarities according to the global distribution of data points. The EDT can be interpreted either as deformation of the feature space or as the result of emergent interactions among all sample points. Specifically, we devised a probe to detect local "tension," or the force field due to ambient sample points, in a deformed feature space. On a global scale, the EDT is able to change the topology of original Euclidean feature space into a compact sphere. Furthermore, iterating the EDT produces a discrete-time dynamical process purely driven by data set geometry. Using carefully designed Gedankenexperimente, we have shown that EDT has the following properties: (1) perspective contraction, (2) cluster condensation, (3) local deformation, and (4) global deformation effects. These properties arise as different facets of the same mathematical transformation and, thus, should be interpreted in a unified manner. The cosine similarity of EDT is akin to distance correlation [14] and measures the similarity of two random vectors obtained from pairwise similarities to all sample points. Properties (1), (2), and (4) can be understood as mutually enhancing the similarity among a subset of points that share common dissimilar points, while property (3) suggests that common similar points can enhance the similarity between "local" or slightly less similar points.

An adjustable regularizer, such as the number of nearest of neighbors in spectral clustering, is able to qualitatively improve an unsupervised learning algorithm. We have shown that spectral clustering [5] using Gaussian RBF kernels may lead to suboptimal clustering even for some easy synthetic data sets. The reason lies in the fact that Gaussian RBF kernels produce a fully connected network: after restricting each node to communicate with only a specified number of nearest neighbors, the resulting similarity network became sparse and the performance of spectral clustering improved. The sequence of iterated EDT indexed by discrete "time" $\tau$ plays a similar role in hierarchical clustering: increasing $\tau$ brings similar sample points into tighter proximity, while enhancing the contrast between clusters (communities). The EDT thus helps hierarchical clustering by utilizing information about the global data distribution. Furthermore, the improvement in clustering accuracy arises from the transformation of data set geometry; thus, any learning algorithm based on pairwise dissimilarity should also benefit from the desirable properties of EDT.

Although the key properties of EDT were first extracted in low feature dimensions in this paper, these advantages, arising from capturing the intrinsic geometry of data distribution, are independent of the feature space dimension, as demonstrated by our finding that EDT also improved the hierarchical clustering of two biological data sets containing 4000 features. 

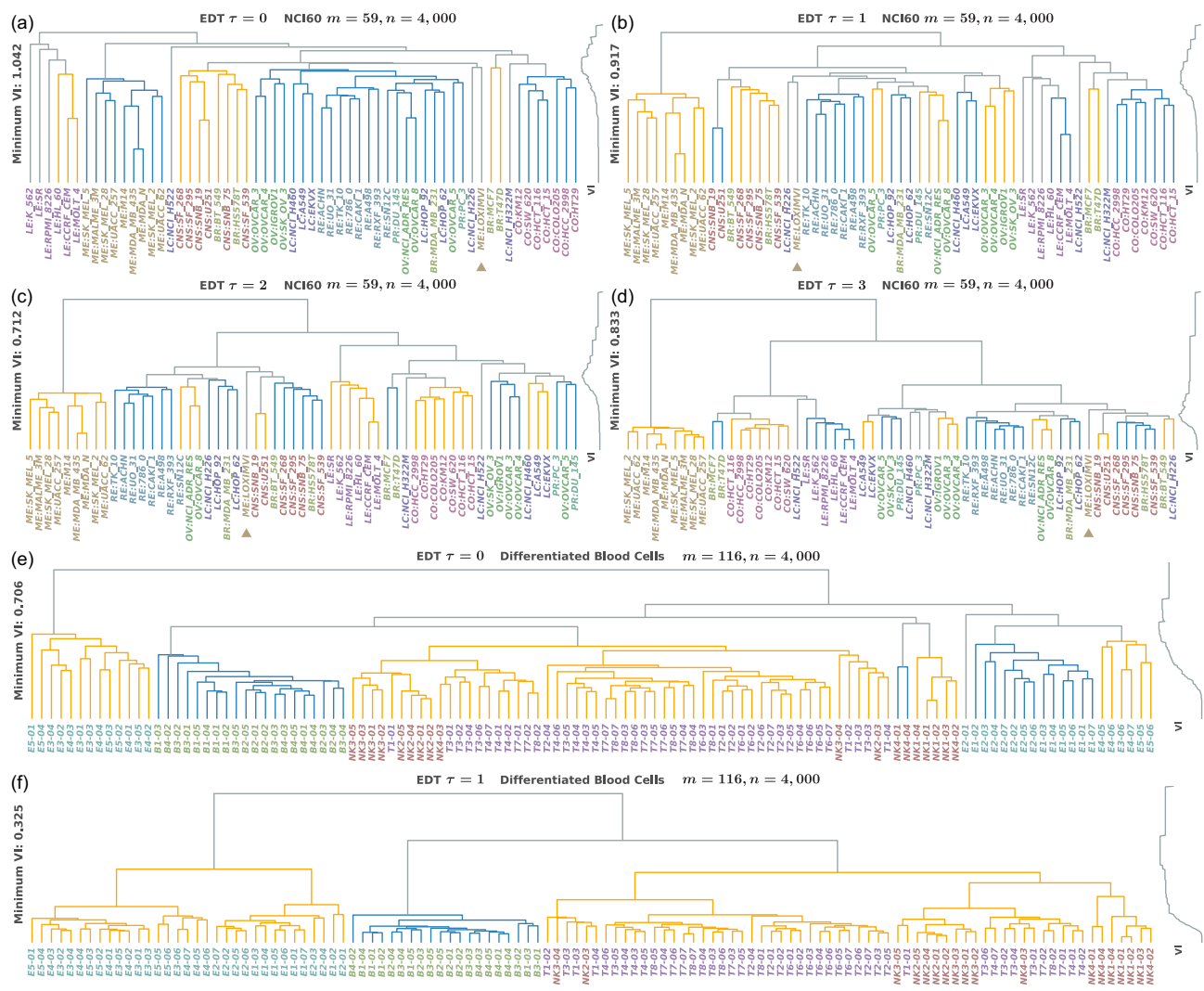

FIG. 10. Hierarchical clustering of NCI60 cancer cell lines [(a)-(d): $m=59$ samples] and human differentiated blood cells [(e),(f): $m=116$ samples] with $n=4000$ most variable genes (with largest standard deviations across all samples) in each data set. For the NCI60 data, the original Euclidean distance [(a): $\tau=0$ ] gave minimum VI of 1.042; but, after two rounds of EDT [(c): $\tau=2$ ], the VI reduced by $31.7 \%$ to 0.712. The original Euclidean distance failed to combine all leukemia (LE) cell lines, but EDT [(c),(d): $\tau=2,3]$ brought LE cell lines together into a single cluster. From the very beginning [(a): $\tau=0$ ], the melanoma cell lines were in a distinct single cluster except for one outlier LOXIM-VI, which is a desmoplastic melanoma cell line and is biologically similar to neurofibroma. Among the misclassified cell lines after two iterations of EDT, the LOXIM-VI found itself more similar to the mixture cluster of central nervous system (CNS) and breast cancer (BR) cell lines. (e) For the blood cell data, the original Euclidean distance split the erythrocyte (Ek, where larger values of $k$ indicate latter stages of maturity) samples into several small subclusters, and the VI was 0.706. (f) After one iteration of EDT, the VI reduced by 54.0\% to 0.325 , and all E $k$ samples were grouped into a single cluster with two branches-immature red blood cells (E1, E2) and more mature blood cells (E3, E4, E5)—well separated from the immune cells: T-cells, B-cells, and natural killer (NK) cells. These results support that the EDT can help improve clustering performance in real data analysis.

As an additional verification of the robustness of EDT in high feature dimensions, our simulation shows that the EDT helps increase the contrast in dissimilarity of bimodal Gaussian clouds even in feature dimensions as high as $10^{3}$, where EDT adapts to the increase in feature dimension by increasing the time index $\tau$ (Appendix B 5).

The EDT was motivated by the multinomial interpretation of a non-negative data vector and its mapping to a hypersphere [15]. In this view, the EDT first takes elementwise square root of the dissimilarity matrix, normalizes each column of $d_{i j}^{\frac{1}{2}}$ by its $L^{2}$-norm, and finally evaluates the cosine dissimilarity between the normalized column vectors lying on a hypersphere. One can consider generalizing this approach by raising the dissimilarity matrix to a different power $\alpha>0$, i.e., taking $d_{i j}^{\alpha}$. Large values of $\alpha$ will have the effect of selectively amplifying large elements in the column vector; in the limit $\alpha \rightarrow \infty$, all but the largest element in each column will be set to zero on the hypersphere. By contrast, small values of $\alpha$ will reduce the contrast between elements in a column; in the limit $\alpha \rightarrow 0$, all normalized column vectors will point in the direction $(1, \ldots, 1,0,1, \ldots, 1)$, forming a single group. Thus clustering will be poor when $\alpha$ is either too small or too large. Nevertheless, tuning $\alpha$ amounts to performing feature selection, and we have evaluated the effects of changing $\alpha$ on the hierarchical clustering of cancer cell lines (NCI60) and human differentiated blood cell (HHC) data (Table I). Comparing the performance of hierarchical clustering without EDT $(\tau=0)$ and with one iteration of EDT

TABLE I. Clustering variation of information as a function of $\alpha$.

\begin{tabular}{lcccccccc}
\hline \hline & & \multicolumn{8}{c}{$\alpha$} \\
\cline { 3 - 9 } & $\tau=0$ & $\frac{1}{20}$ & $\frac{1}{8}$ & $\frac{1}{4}$ & $\frac{1}{2}$ & 1 & 2 & 5 \\
\hline NCI60 & 1.042 & 0.960 & 0.899 & 0.918 & 0.917 & 0.864 & 0.961 & 1.075 \\
HHC & 0.706 & 0.325 & 0.485 & 0.325 & 0.325 & 0.503 & 0.537 & 1.005 \\
\hline
\end{tabular}



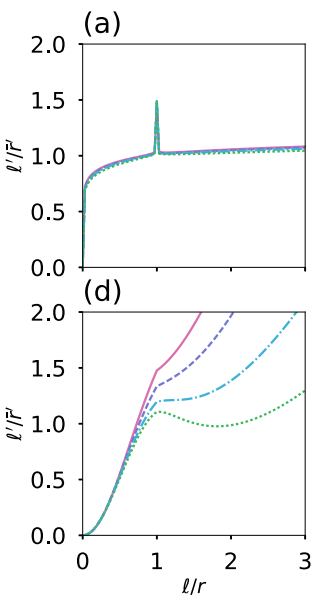

(b)

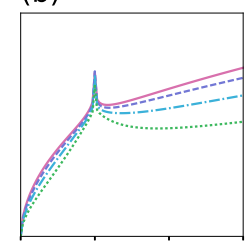

(e)

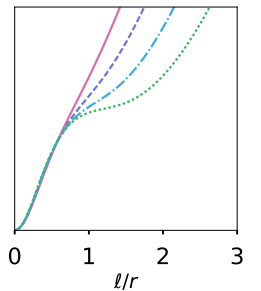

(c)

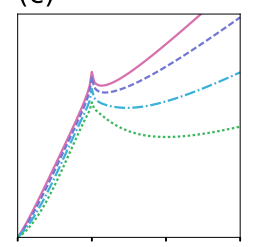

(f)

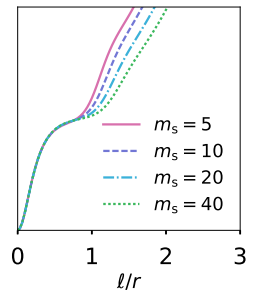

FIG. 11. Outlier absorption effect under different $\alpha$ values. Figures (a)-(f) correspond to $\alpha=\frac{1}{20}, \frac{1}{4}, \frac{1}{2}, 1,2,5$, respectively. For $\alpha>\frac{1}{2}$, the singularity at $\ell / r=1$ was attenuated, and the trapping window was shortened as $\alpha$ increased; by contrast, for $\alpha<\frac{1}{2}$, the singular peak grew sharper as $\alpha$ decreased, and the outlier far from the ideal cluster can still be absorbed into the cluster.

at $\alpha=\frac{1}{20}, \frac{1}{8}, \frac{1}{4}, \frac{1}{2}, 1,2$, or 5 showed that the generalized EDT performed similarly well when $\alpha \approx \frac{1}{2}$, but tended to underperform when $\alpha \gg \frac{1}{2}$ or $\alpha \ll \frac{1}{2}$; however, different data sets may favor different choices of $\alpha$.

In addition, we also tested how modulating $\alpha$ affects the clustering of our synthetic annulus data sets shown in Fig. 8. We found that (1) very large or small values, e.g., $\alpha=5$ or $\frac{1}{20}$, gave poor clustering results, (2) $\alpha=\frac{1}{4}$ gave identical clustering results as $\alpha=\frac{1}{2}$, and (3) $\alpha=1$ failed to cluster the "hard data set" correctly. Similar observations were found in the reanalysis of "single outlier absorption" effect illustrated in Fig. 1(e) for different values of $\alpha$ (Fig. 11): $\alpha$ values greater than 0.5 tended to weaken the absorption effect and shorten the

(a)

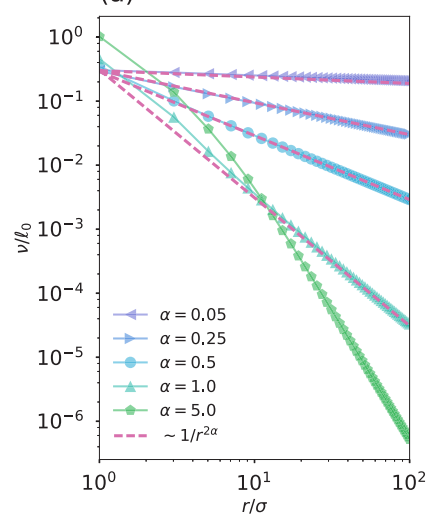

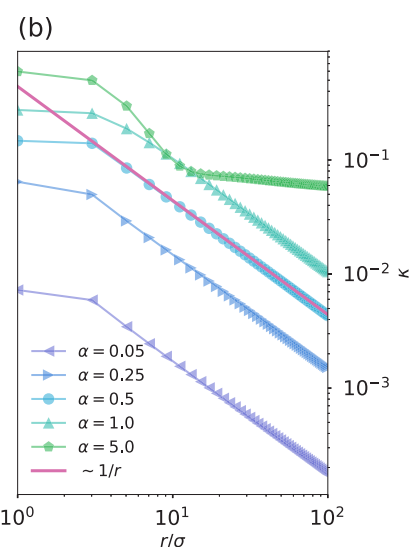

FIG. 12. Effects of generalized EDT. We measured (a) the volume effect captured by $v$ and (b) anisotropy $\kappa$ of a bivariate Gaussian cloud $\mathcal{N}\left((0,0)^{\top}, \sigma^{2}=0.01\right)$ of 50 samples in a plane, using the detector shown in Fig. 1(d). The original EDT $\left(\alpha=\frac{1}{2}\right)$ showed $1 / r$ power law decay, where $r$ is the distance between the center of Gaussian cloud and center of the detector. For $\alpha \gg \frac{1}{2}$, both volume effect and anisotropy deviated from simple power laws. trapping range, but values smaller than 0.5 tended to amplify the absorption.

To investigate the effect of $\alpha$ further, we generated a bivariate Gaussian cloud $\mathcal{N}\left((0,0)^{\top}, \sigma^{2}=0.01\right)$ of 50 samples, and benchmarked the volume effect captured by $v$ and anisotropy captured by $\kappa$ using the detector shown in Fig. 1(d) (Fig. 12). Denoting the distance between the center of bivariate Gaussian distribution and the detector by $r$, our original EDT ( $\alpha=\frac{1}{2}$ ) showed $\sim 1 / r$ power law decay for both $\nu$ and $\kappa$. Increasing (decreasing) $\alpha$ induced faster (slower) power law decay of $v$, but the $\kappa$ anisotropy robustly followed $\sim 1 / r$ for a range of moderate values of $\alpha$. Near $\alpha \approx \frac{1}{2}$, we observed that $v$ followed a power law described by $\sim 1 / r^{2 \alpha}$. Both $v$ and $\kappa$ showed significant deviations from the original EDT when $\alpha \gg \frac{1}{2}$. Therefore, within moderate values of $\alpha$, one can control the volume effect by tuning the power $\alpha$, where smaller $\alpha$ implies slower decay.

\section{ACKNOWLEDGMENTS}

We thank Alex Finnegan and $\mathrm{Hu}$ Jin for critical reading of the manuscript and helpful comments. We also thank the anonymous reviewers for their helpful suggestions. This research was supported by a Distinguished Scientist Award from the Sontag Foundation and the Grainger Engineering Breakthroughs Initiative.

\section{APPENDIX A: DATA PREPARATION}

Two public data sets were used in the hierarchical clustering analysis: (1) NCI60 gene expression data in 59 cancer cell lines comprising nine cancer types, and (2) 116 differentiated blood cell samples in four cell types from human hematopoietic cell (HHC) gene expression data [17]. The nine cancer types in NCI60 data were five breast (BR), six central nervous system (CNS), seven colon (CO), six leukemia (LE), 10 melanoma (ME), eight nonsmall cell lung (LC), seven ovarian (OV), two prostate (PR), and eight renal (RE) cancer. The four cell types in the HHC data were red blood cells or erythrocytes (E), T-cells (T), B-cells (B), and natural killer cells (NK). For both data sets, approximately 4000 most variable genes were selected as features.

Samples from each data set were first clustered with the usual Euclidean distance $d_{i j}^{(0)}=\left\|\mathbf{x}_{i}-\mathbf{y}_{j}\right\|^{2}$, and then with the EDT dissimilarity $d_{i j}^{(\tau)}$ computed from $d_{i j}^{(0)}$. Average linkage was used in all hierarchical clustering analysis, unless indicated otherwise. To quantify the clustering performance unambiguously, the minimum distance from a given clustering to the standard reference clustering was found by measuring the variation of information (VI), which is a well-defined metric function that computes the distance between different partitions (clusterings) of a given set [7]. The reference clustering for NCI60 was the known nine cancer types (BR, CNS, CO, LE, ME, LC, OV, PR, and RE); the reference clustering for HHC was the known four cell types $(\mathrm{E}, \mathrm{T}, \mathrm{B}$, and NK). 


\section{APPENDIX B: EFFECTIVE DISSIMILARITY TRANSFORMATION}

The following properties of EDT mentioned in the main article were obtained from the Gedankenexperimente illustrated in Figs. 2(b)-2(f).

\section{Perspective contraction}

The three points $\left\{P_{1}, P_{2}, P_{3}\right\}$ shown in Fig. 1(b) form two distinct configurations: (1) aligned in a line and (2) forming a triangle in a plane. For case (1), let $P_{1}$ and $P_{2}$ be at $x=+a / 2$ and $-a / 2$, respectively, and $P_{3}$ at $x=b>a / 2$. Then, the original dissimilarity matrix is

$$
d^{(0)}=\left(\begin{array}{ccc}
0 & a & b+\frac{1}{2} a \\
a & 0 & b-\frac{1}{2} a \\
b+\frac{1}{2} a & b-\frac{1}{2} a & 0
\end{array}\right),
$$

and the transformed feature vectors are

$$
\begin{aligned}
& \hat{p}_{1}=\frac{1}{\sqrt{b+\frac{3}{2} a}}\left(\begin{array}{c}
0 \\
\sqrt{a} \\
\sqrt{b+\frac{1}{2} a}
\end{array}\right), \\
& \hat{p}_{2}=\frac{1}{\sqrt{b+\frac{1}{2} a}}\left(\begin{array}{c}
\sqrt{a} \\
0 \\
\sqrt{b-\frac{1}{2} a}
\end{array}\right),
\end{aligned}
$$

and

$$
\hat{p}_{3}=\frac{1}{\sqrt{2 b}}\left(\begin{array}{c}
\sqrt{b+\frac{1}{2} a} \\
\sqrt{b-\frac{1}{2} a} \\
0
\end{array}\right)
$$

From these feature vectors, we compute the first EDT dissimilarity matrix components to be

$$
\begin{gathered}
d_{12}^{(1)}=1-\hat{p}_{1} \cdot \hat{p}_{2}=1-\sqrt{\frac{b-\frac{1}{2} a}{b+\frac{3}{2} a}}, \\
d_{13}^{(1)}=1-\hat{p}_{1} \cdot \hat{p}_{3}=1-\sqrt{\frac{a\left(b-\frac{1}{2} a\right)}{2 b\left(b+\frac{3}{2} a\right)}},
\end{gathered}
$$

and

$$
d_{23}^{(1)}=1-\hat{p}_{2} \cdot \hat{p}_{3}=1-\sqrt{\frac{a}{2 b}} .
$$

As $b / a \uparrow \infty$, we have $d_{12}^{(1)} \downarrow 0, d_{13}^{(1)} \uparrow 1$, and $d_{23}^{(1)} \uparrow 1$; in other words, the EDT ruler length will shrink to zero if the observer moves away from the ruler. Next, we can calculate the relative dissimilarity, i.e., the observed difference between $P_{1}$ and $P_{2}$ from the perspective of $P_{3}$ measured in units of the transformed dissimilarity $d_{12}^{(1)}$, to be

$$
\frac{d_{13}^{(1)}-d_{23}^{(1)}}{d_{12}^{(1)}}=\sqrt{\frac{a}{2 b}} .
$$

Therefore, as the observer moves away from the ruler, the EDT ruler length shrinks to zero, but the observed difference shrinks even faster. In the application of hierarchical clustering, the diminishing difference between the nearest $\left(P_{2}\right)$ and the farthest $\left(P_{1}\right)$ point with respect to the outlier $P_{3}$ implies that clustering derived from the EDT tends to be robust against the choice of linkage, which may be single (nearest point), average, or complete (farthest point).

For case (2), we set up a Cartesian coordinate system in $\mathbb{R}^{2}$ such that $P_{1}, P_{2}$, and $P_{3}$ are located at $(0, a / 2),(0,-a / 2)$, and $(b, 0)$, respectively, where we assume $a, b>0$. The original Euclidean distance matrix is thus

$$
d^{(0)}=\left(\begin{array}{lll}
0 & a & c \\
a & 0 & c \\
c & c & 0
\end{array}\right)
$$

where $c=\sqrt{\left(\frac{a}{2}\right)^{2}+b^{2}}$. The transformed feature vectors are

$$
\begin{aligned}
& \hat{p}_{1}=\frac{1}{\sqrt{a+c}}\left(\begin{array}{c}
0 \\
\sqrt{a} \\
\sqrt{c}
\end{array}\right), \\
& \hat{p}_{2}=\frac{1}{\sqrt{a+c}}\left(\begin{array}{c}
\sqrt{a} \\
0 \\
\sqrt{c}
\end{array}\right),
\end{aligned}
$$

and

$$
\hat{p}_{3}=\frac{1}{\sqrt{2}}\left(\begin{array}{l}
1 \\
1 \\
0
\end{array}\right)
$$

The corresponding transformed dissimilarity matrix elements are

$$
d_{12}^{(1)}=1-\hat{p}_{1} \cdot \hat{p}_{2}=1-\frac{c}{a+c}
$$

and

$$
d_{23}^{(1)}=d_{13}^{(1)}=1-\hat{p}_{1} \cdot \hat{p}_{3}=1-\sqrt{\frac{1}{2} \frac{a}{a+c}} .
$$

As $b / a$ increases to infinity, $d_{12}^{(1)}$ monotonically decreases to zero. Thus the effective ruler length $d_{12}$ approaches zero from the perspective of point $P_{3}$ as it moves far away.

\section{Cluster condensation}

When clustering real data sets, the contrast between the intercluster distance and the intracluster variance is often not very dramatic, making it very difficult to separate the clusters. Therefore, if the data points could condense to the respective centroid locations, then it would improve clustering accuracy considerably; this effect is precisely what EDT accomplishes. For the synthetic data shown in Fig. 1(c), the EDT centroid-centroid dissimilarity $d_{\mathrm{cc}}^{(1)}$ increased relative to the average centroid-satellite distance $\left\langle d_{\mathrm{cs}}^{(1)}\right\rangle$, or the contrast captured by the ratio $d_{\mathrm{cc}}^{(1)} /\left\langle d_{\mathrm{cs}}^{(1)}\right\rangle$, grew more rapidly than $d_{\mathrm{cc}}^{(0)} / d_{\mathrm{cs}}^{(0)}$. Moreover, for fixed $d_{\mathrm{cc}}^{(0)} / d_{\mathrm{cs}}^{(0)}$, increasing the number of satellites $m_{\mathrm{s}}$ around each centroid amplified the contrast ratio [Fig. 2(a)]. 
Throughout the simulations, we did not use any information about the cluster labels, and the improvement of contrast is purely driven by the data. The dense clusters condense while pushing themselves away from other clusters. In other words, within a cluster, the EDT acts similar to gravity, whereas the transformation inflates the space between clusters.

\section{Local deformation}

In Fig. 1(e), $r$ denotes the radius of the cluster and $\ell$ the distance between a single test point and the cluster centroid. We simulated the effect of increasing $\ell$ on the EDT. As $\ell / r \downarrow 1$, we observed a window where the transformed ratio $\ell^{\prime} / \bar{r}^{\prime}$ was less than or equal to the local peak at $\ell / r=1$ [Fig. 2(b)]. This phenomenon can be interpreted as the cluster's trying to reabsorb the test point that is escaping to become an outlier. We also observed that the range of absorption window increased as the cluster size $m_{\mathrm{s}}$ increased, thus making it easier for an outlier to tunnel back to a denser cluster [Fig. 2(b)]. Moreover, the test point also deformed the shape of the cluster, and the satellite points on the circle acted like an elastic membrane that trapped the test point and hindered it from escaping the cluster through elongation.

\section{Global deformation}

Consistent with the single test point example, the cluster in Fig. 1(f) tended to attract the two escaping outliers, as manifested by the fact that as $m_{\mathrm{s}}$ increased, the ratio $\ell^{\prime} / r^{\prime}$ decreased [Fig. 2(c)]. Counterintuitively, $\ell^{\prime} / \ell_{\frac{1}{2}}^{\prime}$ also dropped below 1 as $\ell / r$ increased [Fig. 2(d)]; that is, the two test points became more similar as they departed from the cluster centroid in opposite directions. This paradox can be resolved by merging the points at infinity to a single point, or by topologically transforming the Euclidean space into a hypersphere. We explicitly demonstrated our idea using two circularly distributed data sets shown in Fig. 7. We first observed that (a)

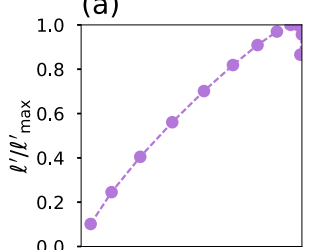

(d)

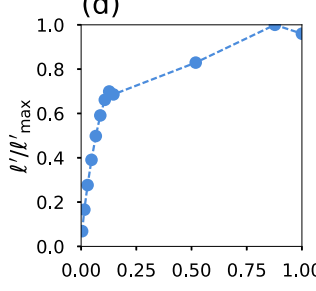

(b)

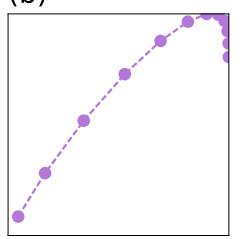

(e)

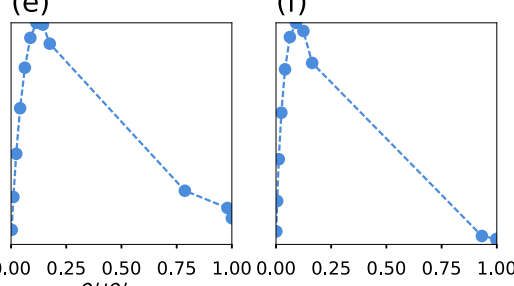

(c)

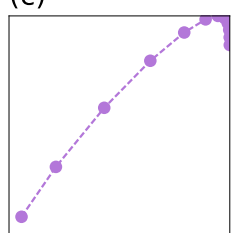

\section{(f)} $\theta^{\prime} / \theta_{\text {max }}^{\prime}$
FIG. 13. Plots of the empirical function $f$ that satisfies $\ell^{\prime}=$ $f\left(\theta^{\prime}\right) \ell_{\max }^{\prime}$, where $\ell^{\prime}$ is the EDT dissimilarity between two neighboring points on a circle and $\theta^{\prime}$ is the EDT dissimilarity between the centroid and the circle. The three plots on the top (a)-(c) correspond to the top three spheres in Fig. 7, and similarly for the three plots on the bottom (d)-(f). the effective dissimilarity between two neighboring points in the outer rings shrank faster than that between neighboring points in the inner rings. To better visualize this phenomenon, we then displayed the dissimilarities on a sphere using the following methods.

For a ring of $k$ points distributed on a unit two-sphere at constant colatitude $\theta \in[0, \pi]$ and uniformly partitioned longitude $\phi_{i} \in[0,2 \pi], i=1, \ldots, k$, the latitude distance $\ell$ between any two neighboring points is equal to $\sin \theta \delta \phi$, where $\delta \phi=2 \pi / k$. Thus $\ell$ attains its maximum value $\ell_{\max }=\delta \phi$ at the equator $\theta=\frac{\pi}{2}$. Note that regardless of the size of $\delta \phi$, we always have $\ell / \ell_{\max }=\sin \theta$; we will utilize this fact to display the EDT-deformed concentric rings shown in Fig. 7. For this purpose, it might appear natural to identify the centroid as the north pole of the sphere, and then identify the colatitude $\theta^{\prime}$ of a ring as the EDT dissimilarity between the centroid and a point on the ring. However, while the distance between two neighboring data points on the sphere at such $\theta^{\prime}$ would then be fixed to be $\sin \theta^{\prime} \delta \phi$, the actual EDT dissimilarity $\ell^{\prime}$ might be different. We thus empirically calculated the function $f\left(\theta^{\prime}\right)$ that satisfies $\ell^{\prime}=f\left(\theta^{\prime}\right) \ell_{\max }^{\prime}$. We then used the location $\theta_{\frac{\pi}{2}}$ of the global maximum of $f$ to calibrate the equator location, and

(a)

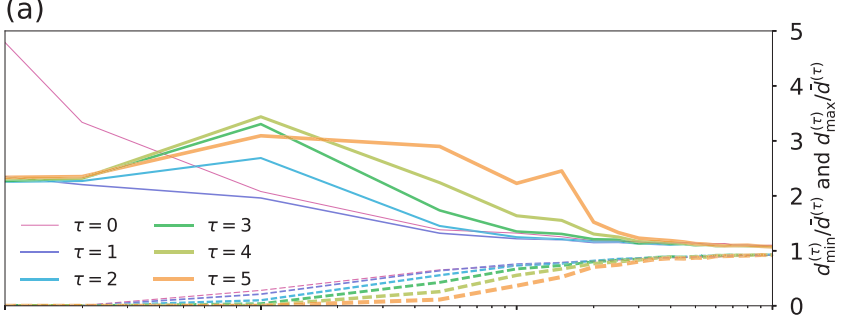

(b)

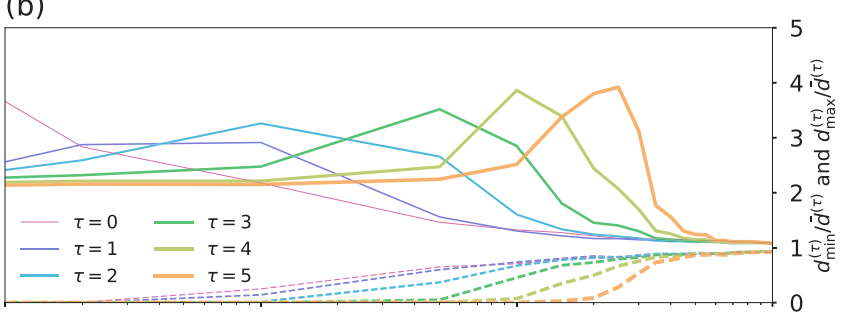

(c)

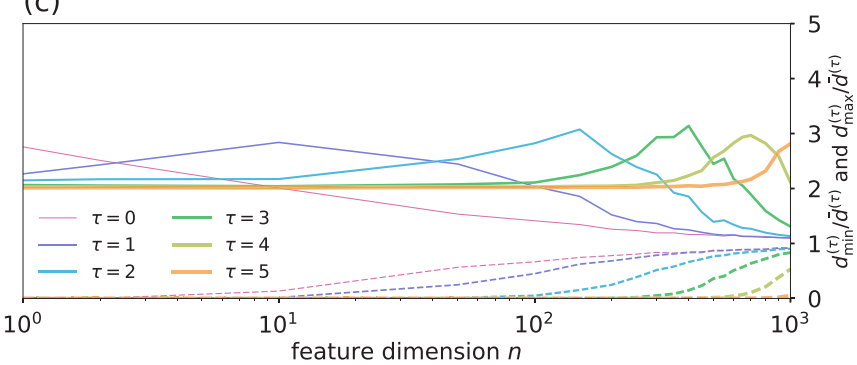

FIG. 14. Plots of maximum and minimum dissimilarities normalized by mean dissimilarity: $d_{\max }^{(\tau)} / \bar{d}^{(\tau)}$ (solid) and $d_{\min }^{(\tau)} / \bar{d}^{(\tau)}$ (dashed) of two multivariate normal distributions $\mathcal{N}\left(( \pm \ell / 2,0, \ldots, 0,0)^{t}, \sigma^{2} I_{n}\right)$ in $\mathbb{R}^{n}$ with variations in (a) $\ell / \sigma=0$, (b) $\ell / \sigma=4$, and (c) $\ell / \sigma=10$. For all three cases (a)-(c), EDT $(\tau>0)$ enlarged the difference between $d_{\max }^{(\tau)} / \bar{d}^{(\tau)}$ and $d_{\min }^{(\tau)} / \bar{d}^{(\tau)}$, and hence enhanced the contrast; when the initial intercluster distance $\ell \gg \sigma$, EDT with high index $\tau$ preserved contrast dramatically relative to initial Euclidean distance $d^{(0)}$, consistent with cluster condensation effect of EDT. 
then calculated the effective colatitude $\tilde{\theta}$ defined as

$$
\tilde{\theta}= \begin{cases}\arcsin \frac{\ell^{\prime}}{\ell_{\max }^{\prime}}, & \theta^{\prime} \leqslant \theta_{\frac{\pi}{2}}, \\ \pi-\arcsin \frac{\ell^{\prime}}{\ell_{\max }^{\prime}}, & \theta^{\prime}>\theta_{\frac{\pi}{2}}\end{cases}
$$

to display the concentric rings on the sphere, as shown in Fig. 7. Figure 13 shows the $f\left(\theta^{\prime}\right)$ for the two circular data sets shown in Fig. 7 after $\tau$ iterations of EDT.

\section{EDT and the curse of dimensionality}

The loss of contrast in Euclidean distance is one of the symptoms of the curse of dimensionality; to be exact, the longest distance $d_{\max }^{(0)}$ and shortest distance $d_{\min }^{(0)}$ between any pair of points in a data set will both asymptotically approach the mean distance $\bar{d}^{(0)}$ in the large feature dimension limit $n \uparrow \infty$. To see whether EDT can help improve the contrast between clusters in high dimensions, we simulated two $n$-dimensional Gaussian distributions $\mathcal{N}\left(( \pm \ell / 2,0, \ldots, 0,0)^{\top}, \sigma^{2} I_{n}\right), 100$ points from each, for $\ell / \sigma=0,4$, and 10 . We then computed the Euclidean distance matrix $d^{(0)}$ and subsequent effective dissimilarity matrices $\left\{d^{(\tau)}\right\}_{\tau=0}^{5}$. Figure 14 shows the normalized pairwise maximum $\left(d_{\max }^{(\tau)} / \overline{\bar{d}}^{(\tau)}\right)$ and minimum $\left(d_{\min }^{(\tau)} / \bar{d}^{(\tau)}\right)$ distance between data points in each dimension. The difference $\left(d_{\max }^{(\tau)}-d_{\min }^{(\tau)}\right) / \bar{d}^{(\tau)}$ generally became larger as the EDT index $\tau$ increased, and the improvement in contrast over the original Euclidean distance in high dimensions was very dramatic when $\ell \gg \sigma$, as seen for $n=1000$ in Fig. 14(c).
[1] L. Kaufman and P. J. Rousseeuw, in Finding Groups in Data, edited by L. Kaufman and P. J. Rousseeuw, An Introduction to Cluster Analysis (John Wiley \& Sons, Hoboken, NJ, 2009).

[2] T. Hastie, R. Tibshirani, and J. Friedman, The Elements of Statistical Learning, Data Mining, Inference, and Prediction (Springer Science \& Business Media, New York, 2013).

[3] G. Paliouras, V. Karkaletsis, and C. D. Spyropoulos, Machine Learning and Its Applications, Advanced Lectures (Springer, New York, 2003).

[4] U. Von Luxburg, Stat. Comput. 17, 395 (2007).

[5] A. Y. Ng, M. I. Jordan, and Y. Weiss, in Advances in Neural Information Processing Systems, edited by T. G. Dietterich, S. Becker, and Z. Ghahramani, On Spectral Clustering: Analysis and an algorithm (MIT Press, Cambridge, MA, 2001).

[6] R. R. Coifman and S. Lafon, Appl. Comput. Harmon. Anal. 21, 5 (2006).

[7] M. Meilă, J. Multivar. Anal. 98, 873 (2007).

[8] H.-J. Li, Y. Wang, L.-Y. Wu, J. Zhang, and X.-S. Zhang, Phys. Rev. E 86, 016109 (2012).

[9] J. Reichardt and S. Bornholdt, Phys. Rev. Lett. 93, 218701 (2004).
[10] A. Zimek, E. Schubert, and H.-P. Kriegel, Stat. Anal. Data Mining: ASA Data Sci. J. 5, 363 (2012).

[11] K. P. Bennett, U. Fayyad, and D. Geiger, Density-based Indexing for Approximate Nearest-Neighbor Queries (ACM, New York, 1999).

[12] M. E. Houle, H.-P. Kriegel, P. Kröger, E. Schubert, and A. Zimek, in Scientific and Statistical Database Management (Springer, Berlin, 2010), pp. 482-500.

[13] P. W. Anderson, Science 177, 393 (1972).

[14] G. J. Székely, M. L. Rizzo, and N. K. Bakirov, Ann. Stat. 35, 2769 (2007).

[15] J. Lafferty and G. Lebanon, J. Machine Learning Res. 6, 129 (2005).

[16] C. Zhao and J. S. Song, arXiv:1702.01373.

[17] N. Novershtern, A. Subramanian, L. N. Lawton, R. H. Mak, W. N. Haining, M. E. McConkey, N. Habib, N. Yosef, C. Y. Chang, T. Shay, G. M. Frampton, A. C. B. Drake, I. Leskov, B. Nilsson, F. Preffer, D. Dombkowski, J. W. Evans, T. Liefeld, J. S. Smutko, J. Chen, N. Friedman, R. A. Young, T. R. Golub, A. Regev, and B. L. Ebert, Cell 144, 296 (2011).

[18] I. Yeh and T. H. McCalmont, J. Cutaneous Pathol. 38, 625 (2011). 